Check for updates

Cite this: RSC Adv., 2018, 8, 29980

\title{
Preparation methods and release kinetics of Litsea cubeba essential oil microcapsules
}

\begin{abstract}
Yan-hong Yang, (D) ${ }^{a}$ Xiang-zhou Li ${ }^{\star a b}$ and Sheng Zhang ${ }^{a}$
In this paper, using $\beta$-cyclodextrin ( $\beta-C D)$ as the shell material, LCEO (Litsea cubeba essential oil) microcapsules were prepared by various preparation methods, such as grinding, saturated solution, freeze-drying and spray-drying. The encapsulation yield, encapsulation efficiency, retention rate of the microcapsules and the citral content of the microcapsules were investigated. The surface morphologies of the microcapsules were observed using SEM (Scanning Electronic Microscopy); the entrapment efficiencies of the microcapsules were detected using IR (Infrared Spectrum) analysis; the citral contents of microcapsules were detected by GC (Gas Chromatography) analysis. The highest encapsulation efficiency for the microcapsules was obtained using spray-drying, followed by freeze-drying, saturated aqueous solution and grinding, while the encapsulation yield followed the opposite sequence to the encapsulation efficiency. At a specific storage temperature $\left(15^{\circ} \mathrm{C}\right)$ and humidity $(60 \%)$, spray-drying had the most satisfactory protective effect on citral in LCEO, followed by freeze-drying and saturated aqueous solution, while the grinding method appeared to provide the worst protective effect. Avrami's model was used to simulate the release rates of the four kinds of microcapsules. The release mechanism parameters of microcapsules prepared by grinding, saturated aqueous solution, freeze-drying and spraydrying were $0.961,1.096,1.156$ and 0.945 , respectively. The release rate constants of microcapsules prepared by grinding, saturated aqueous solution, freeze-drying and spray-drying were $2.53 \times 10^{-2}, 2.22$ $\times 10^{-2}, 1.84 \times 10^{-2}$, and $7.27 \times 10^{-3} \mathrm{~d}^{-1}$, respectively. It was concluded that the release reactions of the microcapsules prepared by grinding or spray-drying lay between the diffusion limiting kinetics and the first-order release kinetics, and the release reactions of the microcapsules prepared by saturated

aqueous solution or freeze-drying were larger than the first-order release kinetics.
\end{abstract}

Received 6th July 2018

Accepted 6th August 2018

DOI: $10.1039 / c 8 r a 05769 a$

rsc.li/rsc-advances

\section{Introduction}

Litsea cubeba (Lour) Pers. is a specific perfume-producing plant in South China. LCEO is extracted from the fruit of Litsea cubeba. The main component of LCEO is citral (neral and geranial). ${ }^{1}$ Citral, usually colorless or a pale yellow liquid with an intensely lemon-like and spicy aroma, could be used as a significant raw material in the perfume and pharmaceutical industries. ${ }^{2-4}$ In addition, citral has also been found to have bioactivities such as anti-inflammatory, antimicrobial, antioxidant, pesticidal and anticancer properties. ${ }^{\mathbf{5} 6}$ Most essential oils like LCEO are sensitive materials, ${ }^{7}$ which can easily suffer degradation under the action of light, oxygen and moderate temperatures. Relatively poor stability has prevented their longterm applications.

Microencapsulation is a technology that uses membranous materials to coat solids, liquids or gases into microparticles.

${ }^{a}$ School of Materials Science and Engineering, Central South University of Forestry and Technology, Changsha, Hunan, P. R. China.E-mail: rlxz@163.com; csyyh_69@163. com; Fax: +86-0731-8562-3303; Tel: +86-0731-8562-3309

${ }^{b}$ State Key Laboratory of Ecological Applied Technology in Forest Area of South China, Changsha, Hunan, P. R. China
And microencapsulation technology has significant effects on the protection of plant essential oils, improving the stability of essential oils and their controlled release. ${ }^{8-11}$ Therefore, the selection of shell materials is particularly important. $\beta$-CD is an enzymatically modified starch, shaped like a hollow cone, which has been widely used for the encapsulation of essential oils, giving some of the highest encapsulation yields, due to the formation of inclusion complexes between the oil and the apolar cavity of CD. ${ }^{12}$ Thus $\beta$-CD was used as the shell material in this paper.

Encapsulation techniques can be divided into three classes: physical processes, chemical processes and physicochemical processes. However, the preparation technique for microcapsules with $\beta-C D$ as the shell material is usually a physical process. Common physical methods include grinding, saturated aqueous solution, freeze-drying and spray-drying. The grinding process is simple, but its embedding effect is limited. ${ }^{\mathbf{1 3}}$ The equipment for saturated aqueous solution is universal, but the properties of the shell material limit the microcapsules' embedding effect. ${ }^{14}$ The freeze-drying method ${ }^{12}$ and the spraydrying method ${ }^{15}$ have relatively high encapsulation efficiency, but special equipment is needed, and the costs are relatively 
high. Therefore, it was necessary to conduct a comparative study on the above four encapsulation methods.

At present, there have been a lot of reports on research into a single-technology preparation process for microcapsules. ${ }^{\mathbf{1 6}-18}$ However, a comprehensive and systematic comparison of several preparation techniques is relatively rare. ${ }^{19-21}$ Meanwhile, the release of the core material of the microcapsules determined the application prospects of the microcapsules. In general, the rate of release of the core material was determined by the solubility of the core material in the shell material and the diffusion of the shell material., ${ }^{7,22}$ There have been many release kinetic models, such as Avrami's model, ${ }^{23,24}$ the zeroorder release kinetic equation, ${ }^{25,26}$ the first-order release kinetic equation ${ }^{27,28}$ and the Higuchi model. ${ }^{29,30}$ At present, there are many reports on the release kinetics of essential oil microcapsules. However, theoretical studies on the effect of the rate control of the preparation process on the release process are relatively few.

In order to compare inclusion and release efficiency, $\beta$-CD was used as the shell material, and LCEO microcapsules prepared by four kinds of physical methods: grinding, saturated aqueous solution, freeze-drying and spray-drying were investigated. Avrami's equation was used to fit the release data of the four types of microcapsules and the release mechanism parameters and release rate constants of the microcapsules were obtained. The release reaction mechanism of the microcapsules was analyzed. The quantitative research results obtained by this thesis provided a basis for more effective control over the release of the core material.

\section{Materials and methods}

\section{Materials}

The standard oil (citral content $=96.59 \%$ ) was purchased from Sigma (America); LCEO (citral content $=92.69 \%$ ) was purchased from Yongshun Source Plant Natural Flavors Limited Liability Company (P. R. China); $\beta$-CD and alcohol were supplied by Shanghai Luzhong Chemical Reagent Co. Ltd (P. R. China).

\section{GC analysis of oil}

The standard oil and the LCEO extracted from the microcapsules were analyzed by GC using an Agilent 7890B GC Series under the following conditions: column HP-5 $(30 \mathrm{~m} \times 320 \mu \mathrm{m}$ $\times 0.25 \mu \mathrm{m})$, injector temperature $250{ }^{\circ} \mathrm{C}$; detector temperature $250{ }^{\circ} \mathrm{C}$; programmed column temperature: initial temperature $80{ }^{\circ} \mathrm{C}$ and hold 1 minute, Ramp1: from $80{ }^{\circ} \mathrm{C}$ to $120{ }^{\circ} \mathrm{C}$ at $10{ }^{\circ} \mathrm{C} \min ^{-1}$ and hold 1 minute, Ramp2: from $120^{\circ} \mathrm{C}$ to $150{ }^{\circ} \mathrm{C}$ at $3{ }^{\circ} \mathrm{C} \mathrm{min}{ }^{-1}$ and hold 1 minute, Ramp3: from $150{ }^{\circ} \mathrm{C}$ to $230^{\circ} \mathrm{C}$ at $15^{\circ} \mathrm{C} \min ^{-1}$ and hold 2 minutes. The sample volume was 0.5 $\mu \mathrm{L}$, the split ratio was $50: 1$, and the carrier gas flow rate was 1 $\mathrm{mL} \min ^{-1}$.

\section{Preparation of microencapsulation by grinding}

Three grams of $\beta$-CD powder was packed into a dry mortar, then $1 \mathrm{~mL}$ of LCEO was added dropwise into the mortar and ground for $40 \mathrm{~min}$. The mixture was dried at room temperature and stored in airtight glass containers in the refrigerator prior to analysis.

\section{Preparation of microencapsulation by saturated solution}

Three grams of $\beta$-CD powder was dissolved completely in deionized water at $70{ }^{\circ} \mathrm{C}$, and then $1 \mathrm{~mL}$ of LCEO (dissolved in $1 \mathrm{~mL}$ of alcohol) was added dropwise into the solution. The mixture was stirred continuously for $5 \mathrm{~h}$ and was kept in a refrigerator at about $4{ }^{\circ} \mathrm{C}$ for $12 \mathrm{~h}$. The cold precipitate was recovered by vacuum filtration and dried at $50{ }^{\circ} \mathrm{C}$ (in a roaster) until it achieved constant mass. Microcapsules were stored in airtight glass containers in the refrigerator prior to analysis.

\section{Preparation of microencapsulation by freeze-drying}

Three grams of $\beta$-CD powder was dissolved completely in deionized water at $70{ }^{\circ} \mathrm{C}$, and then $1 \mathrm{~mL}$ of LCEO (dissolved with $1 \mathrm{~mL}$ of alcohol) was added dropwise into the solution. The mixture was stirred continuously for $5 \mathrm{~h}$ and was recovered by vacuum filtration. The residue was frozen at $-18{ }^{\circ} \mathrm{C}$ for $24 \mathrm{~h}$, then dried at $-30{ }^{\circ} \mathrm{C}$ in a freeze dryer (LGJ-10, Beijing) under 76 $\mathrm{kPa}$ for $48 \mathrm{~h}$. The microcapsules were stored in airtight glass containers in the refrigerator prior to analysis.

\section{Preparation of microencapsulation by spray-drying}

Three grams of $\beta$-CD powder was dissolved completely in deionized water at $70{ }^{\circ} \mathrm{C}$, and then $1 \mathrm{~mL}$ of LCEO (dissolved in $1 \mathrm{~mL}$ of alcohol) was added dropwise into the solution. The stirred emulsion was placed in a homogenizer (Panda Plus, GEA Niro Soavi, Italy) at 500 bar for $20 \mathrm{~min}$. The homogenized emulsion was spray dried in a spray dryer (B-290, BUGHI, Switzerland) under the following operational conditions: spray nozzle (inlet) temperature, $180{ }^{\circ} \mathrm{C}$; air inlet volume, $80 \%$; feed pump rate, $15 \%$. The product was collected in a separator and LCEO microcapsules were obtained. The microcapsules were stored in airtight glass containers in the refrigerator prior to analysis.

\section{Determination of surface oil content of microcapsules}

The amount of LCEO on the surface of the microcapsule powder was determined as a measure for the oil released from microcapsules. Two grams of microcapsules were mixed with $15 \mathrm{~mL}$ of petroleum ether, and the mixture was stirred $(2 \mathrm{~min})$ at room temperature. The suspension was then filtered and the residue was washed three times with $20 \mathrm{~mL}$ of petroleum ether. Subsequently the solvent was evaporated under reduced pressure at a temperature of $50{ }^{\circ} \mathrm{C}$. The amount of surface oil was calculated by the difference in the weight of the flask before and after the solvent evaporation. The results were the average of three measurements in each sample.

\section{Determination of total oil content}

Two grams of microcapsules were packed in filter paper and mixed with $150 \mathrm{~mL}$ of petroleum ether, then the mixture was Soxhlet extracted in a water-bath at $85{ }^{\circ} \mathrm{C}$ for $8 \mathrm{~h}$. Subsequently 
the solvent was evaporated under reduced pressure at a temperature of $50{ }^{\circ} \mathrm{C}$. The total amount of oil was calculated by the difference in the weight of the flask before and after the solvent evaporation. The results were the average of three measurements in each sample.

\section{Encapsulation efficiency}

EE (encapsulation efficiency, \%) of the microcapsules, which determines the quantity of oil entrapped into the $\beta$-CD cavity, was calculated with eqn (1):

$$
\mathrm{EE}=\frac{O_{\mathrm{t}}-O_{\mathrm{s}}}{O_{\mathrm{t}}} \times 100
$$

where $O_{\mathrm{t}}, O_{\mathrm{s}}$ are the total mass of oil, and the mass of the surface oil of the microcapsules, respectively.

\section{Encapsulation yield}

EY (encapsulation yield, \%), which determines the quantity of microcapsules, was calculated from eqn (2):

$$
\mathrm{EY}=\frac{M_{\mathrm{a}}}{M_{\mathrm{th}}} \times 100
$$

where $M_{\mathrm{a}}$ is the actual amount of microcapsules and $M_{\mathrm{th}}$ is the theoretical amount of microcapsules.

\section{SEM of microcapsules}

SEM of the microcapsules were obtained using a scanning electron microscope (JEOL 6380-lv, Japan). A small amount microcapsules was scattered evenly onto the surface of an aluminum stub covered with a carbon tab. Excess material was removed using drying compressed air, and the sample was sputter coated with a thin conductive film of gold for approximately 2 min in a sputter coater. The stub containing the coated sample was then placed in the specimen chamber under vacuum. An accelerated electron beam was directed onto the sample and the image was produced based on the backscattered electron beam received. The accelerating voltage used was 15 $\mathrm{kV}$.

\section{Fourier transformation-infrared spectroscopy of microcapsules}

IR spectra of the microcapsules were obtained using a spectrometer (Nicolet Avatar330, America) using $\mathrm{KBr}$ disks. The procedure consisted of grinding the microcapsule (about $5 \mathrm{mg}$ ) together with $\mathrm{KBr}$ (about $100 \mathrm{mg}$ ) into a fine powder, placing the powder into a sampling cup, smoothing the powder and compressing the powder bed into the holder using a compression gauge. The sample was placed in the light path and the spectrum was obtained. The scanning range was maintained from 4000 to $400 \mathrm{~cm}^{-1}$.

\section{Retention of LCEO in the microcapsules during storage}

The stability of encapsulated LCEO during storage was investigated to determine the release characteristics at a temperature of $15{ }^{\circ} \mathrm{C}$ and humidity of $60 \%$. Five gram microcapsules were precisely weighed and spread evenly in a thin layer in a $250 \mathrm{~mL}$ beaker. The beaker was stored in a desiccator and protected from light. The retention of LCEO during release was calculated from eqn (3):

$$
R(\%)=\frac{M_{\mathrm{aL}}}{M_{\mathrm{aT}}} \times 100 \%
$$

where $M_{\mathrm{aL}}$ is the amount of LCEO remaining after a prescribed time and $M_{\mathrm{aT}}$ is the initial amount of LCEO.

\section{Citral content of microcapsules}

The LCEO was extracted from the microcapsules by Soxhlet extraction. The citral content was detected by GC analysis.

\section{Release kinetics of microcapsules}

The release process of the microcapsules might be fitted by Avrami's model, eqn (4):

$$
R=\exp \left[-(k t)^{n}\right]
$$

where $R$ is the retention rate of LCEO in the microcapsules and $t$ is time.

Eqn (5) was obtained by taking the natural logarithm on both sides of eqn (4). According to the experimental data, the linear equation could be fitted with $\ln t$ as the vertical coordinate, and the release mechanism parameter $(n)$ and the release rate constant $(k)$ were obtained from eqn (5):

$$
\ln (-\ln R)=n \ln k+n \ln t
$$

where $n$ is the release mechanism parameter and $k$ is the release rate constant.

\section{Results and discussions}

\section{GC analysis of the standard oil and LCEO}

The citral content of the standard oil and LCEO were detected by GC analysis and are shown in Fig. 1. The citral content is $96.59 \%$ and $92.69 \%$ in the standard oil and total oil, respectively.

\section{Encapsulation efficiency and yield of microcapsules}

The encapsulation efficiency and encapsulation yield of the LCEO microcapsules are shown in Fig. 2. The microcapsules produced using spray-drying had the highest encapsulation efficiency, followed by freeze-drying and saturated aqueous solution, and the embedding rate using grinding was the lowest. The yield followed the opposite sequence to the encapsulation efficiency.

Because the grinding was simple, the process was simple: the preparation process did not go through other instruments, there was no reagent transfer of raw material and its yield was the highest. At the same time, because of the special hydrophobic and externally hydrophilic structure of $\beta-\mathrm{CD}$, a large amount of LCEO was not embedded into the cavity of $\beta$-CD in 

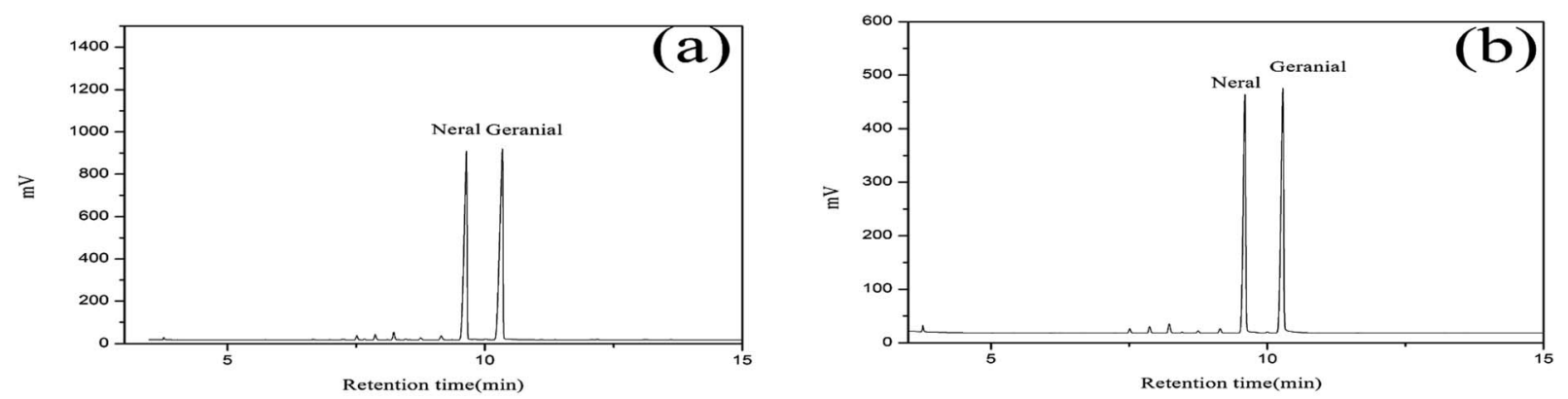

Fig. 1 Gas chromatogram of standard oil (a) and LCEO (b).

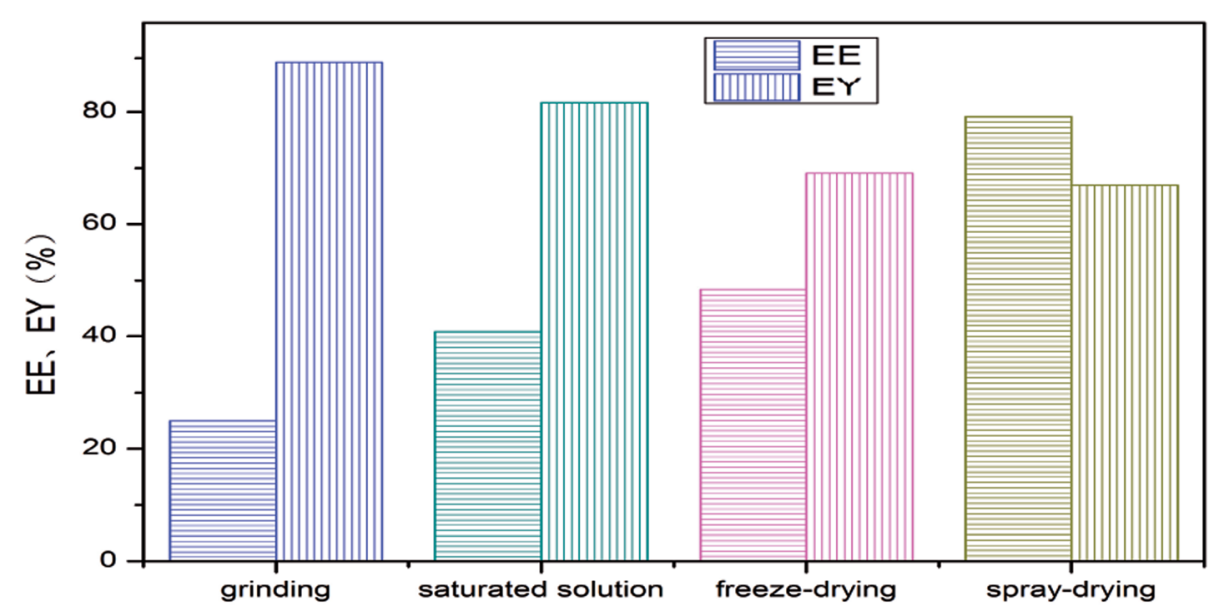

Fig. 2 Encapsulation efficiency and encapsulation yield of microcapsules.

the simple grinding process, so the embedding rate of the prepared microcapsules was the lowest. ${ }^{12}$

However, when the microcapsules were prepared by saturated aqueous solution or freeze-drying, the amount of LCEO embedded in the inner cavity was less due to the low solubility of $\beta$-CD. The loss of LCEO by high-temperature drying of the saturated aqueous solution was slightly higher than for freezedrying, so the embedding rate of the microcapsules was slightly lower than in the freeze-drying method. At the same time, the filtration transfer of microcapsules resulted in a decrease in the yield of microcapsules. ${ }^{13,14,31}$

Compared with the other three methods, spray-drying was more complicated: more instruments and equipment were used, and there was reagent residue in the equipment wall during reagent transfer. The high temperature of spraydrying and the volatilization of LCEO led to the loss of raw materials, so the yield of microcapsules prepared by spraydrying was lower than in the other methods. However, the high pressure of homogenization might promote further diffusion of LCEO into the cavity of $\beta$-CD, so the speed of spray-drying was rapid and the loss of LCEO was small. Therefore, the encapsulation efficiency of microcapsules using spray-drying was higher than for the other three methods. ${ }^{8,15}$

\section{SEM of microcapsules}

Scanning electron micrographs of the microstructures of the microcapsules produced using grinding, saturated aqueous solution, freeze-drying and spray-drying are shown in Fig. 3.

Microcapsules produced using grinding were very uneven (Fig. 3(a)). A lot of LCEO was not embedded in the cavity of the $\beta$-CD but absorbed on the surface. So the microcapsules easily bonded together, causing serious caking.

Microcapsules prepared by saturated aqueous solution had a relatively uneven shape and slight agglomeration (Fig. 3(b)). The microcapsules were not uniform and there was slight caking because of a certain non-uniformity in the operation of the cooling process.

Compared with the previous two types of microcapsules, the size of the microcapsules produced using freeze-drying was relatively uniform (Fig. 3(c)). Stable solid skeletons of material were formed before the sublimation process and remained basically unchanged after sublimation..$^{32}$ So the size of the microcapsules was relatively uniform.

Microcapsules prepared by spray-drying were more uniform than the microcapsules produced using the other three methods (Fig. 3(d)). This was similar to results from the study by Youfeng et al. ${ }^{33}$ The combination of high-pressure homogenization and spray-drying increased degree of uniting of $\beta$-CD and 



Fig. 3 SEM of microcapsules produced using grinding (a), saturated aqueous solution (b), freeze-drying (c) and spray-drying (d).

LCEO and was beneficial to the formation of microcapsules with good compactness and wall strength, ${ }^{34}$ so the encapsulation efficiency of microcapsules produced using spray-drying was higher than that of the other three methods.

\section{IR spectra of microcapsules}

The IR spectra of LCEO, $\beta$-CD and microcapsules produced using grinding, saturated aqueous solution, freeze-drying and spray-drying are shown in Fig. 4 . There are three characteristic absorption peaks in the infrared spectrum of LCEO, the stretching vibration peaks of $-\mathrm{CH}_{3}$ and $-\mathrm{CH}_{2}-$ were 2968,2926 and $2858 \mathrm{~cm}^{-1}$, and the stretching vibration peak of $\mathrm{C}=\mathrm{O}$ was at $1676 \mathrm{~cm}^{-1}$. The stretching vibration peak of $-\mathrm{OH}$ in $\beta$-CD was $3388 \mathrm{~cm}^{-1}$, and there was a stretching vibration peak of $-\mathrm{CH}^{3}$ near $2926 \mathrm{~cm}^{-1}$.

The IR spectra of the microcapsules prepared by grinding showed characteristic absorption peaks of both LCEO and $\beta$ CD. The characteristic peaks had disappeared because of the poor inclusion effect of the grinding and most of the uncovered LCEO was adsorbed on the outer surface of the $\beta$-CD.

There were no peaks of LCEO at 2968 and $2858 \mathrm{~cm}^{-1}$ in the IR spectra of microcapsules produced using saturated aqueous solution, freeze-drying or spray-drying, and the peak intensity of the $\mathrm{C}=\mathrm{O}$ stretching peak at $1676 \mathrm{~cm}^{-1}$ was significantly

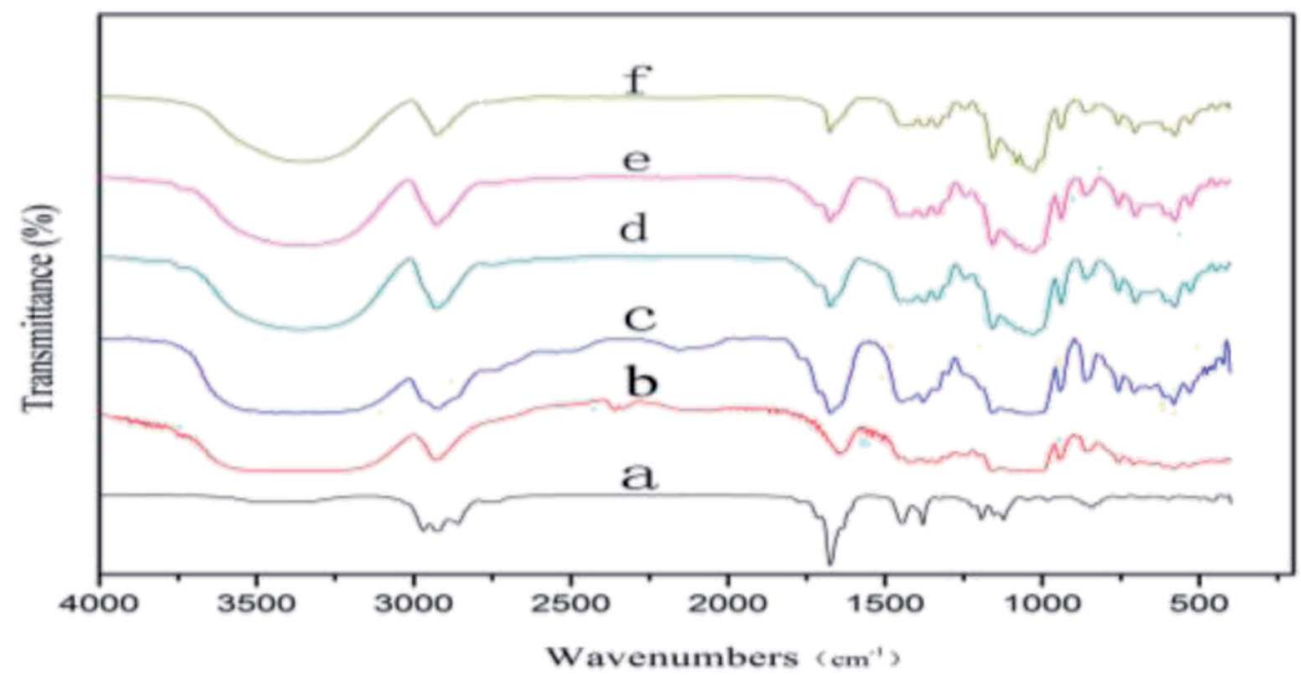

Fig. 4 IR spectra of LCEO (a), $\beta-C D$ (b), microcapsules produced using grinding (c), saturated aqueous solution (d), freeze-drying (e) and spraydrying (f). 




Fig. 5 Retention rates of LCEO and microcapsules.

decreased. More stable inclusion compounds were formed after LCEO entered the cavity of $\beta$-CD because of van der Waals forces, and hydrogen bond forces existing in LCEO and $\beta$-CD. ${ }^{35}$ The combined effect of LCEO and $\beta$-CD microcapsules produced using a high pressure of homogenization was higher than in the other three methods. So the intensity of the characteristic stretching vibration peak of microcapsules produced using spray-drying was weaker than for microcapsules produced using the other three methods.

\section{Retention rate of microcapsules}

The retention rates of the microcapsules were calculated according to eqn (3) and are shown in Fig. 5. The release of LCEO and microcapsules produced using grinding were not greatly different. The microcapsules produced using saturated aqueous solution, freeze-drying or spray-drying had a protective effect on LCEO, which could reduce the loss of LCEO.

The microcapsules produced using grinding had a poor inclusion effect on LCEO because volatilized LCEO adhered to the surface of $\beta$-CD during the release process, so the protection effect of the microcapsules was not obvious. The microcapsules produced using saturated aqueous solution or freeze-drying were mixed under conditions of uniform mixing and stirring. So the inclusion efficiency was not high and the protection of LCEO has certain limitations during the release process. The LCEO of the microcapsules prepared by spray-drying is entrapped into the cavity of the $\beta$-CD by external force under the high pressure of homogenization. So the inclusion efficiency of LCEO was high, which might provide good protection of LCEO. ${ }^{7}$

\section{Citral content of LCEO and microcapsules}

The changes in citral content in the microcapsules during the release process reflected the protective effect of the microcapsules on LCEO. The contents of citral in the microcapsules prepared by spray-drying or freeze-drying were not significantly decreased, while the citral content in the microcapsules prepared by aqueous solution decreased slightly and the content of citral in the microcapsules prepared by grinding was the lowest (Fig. 6). It can be seen that the protective effects of different methods on the citral in LCEO were varied. Spraydrying had the most satisfactory protective effect on the citral in LCEO, followed by freeze-drying and saturated aqueous solution, but grinding had the worst protective effect. These results were consistent with the previous result described above.

\section{Release kinetics of microcapsules}

The microcapsule controlled release system was composed of a core material and a polymer shell material. The controlled release effect was obtained at a temperature of $15{ }^{\circ} \mathrm{C}$ and a humidity of $60 \%$. Avrami's model was used to fit the release process of the microcapsules, and the release mechanism parameter $n$ and the release rate constant $k$ are shown in Table 1.

As shown in Table 1, the release mechanism parameter $n$ of microcapsules prepared by grinding, saturated aqueous solution, freeze-drying and spray-drying were 0.961, 1.096, 1.156 and 0.945 , respectively. The release reaction of the microcapsules prepared by grinding or spray-drying lay between the diffusion limiting kinetics and the first-order release kinetics. The release reaction of the microcapsules prepared by saturated water solution or freeze-drying was greater than that of first-order release kinetics. The release rate constants of microcapsules produced using grinding, saturated aqueous solution, freezedrying and spray-drying were $2.53 \times 10^{-2}, 2.22 \times 10^{-2}, 1.84 \times$ $10^{-2}, 7.27 \times 10^{-3} \mathrm{~d}^{-1}$, respectively. The release rate of microcapsules prepared by grinding was the maximum, followed by microcapsules prepared by saturated solution and freezedrying; the release rate of microcapsules prepared by spraydrying was the lowest. It was shown that spray-drying had the best protective effect on the essential oil, followed by freezedrying and saturated solution, and grinding had the worst protective effect on the essential oil. 




Fig. 6 Citral content of LCEO and microcapsules.

Table 1 The release mechanism parameters $(n)$ and release rate constant $(k)$ of the microcapsules

\begin{tabular}{llll}
\hline & $n$ & $k / \mathrm{d}^{-1}$ & $R^{2}$ \\
\hline Grinding & 0.961 & $2.53 \times 10^{-2}$ & 0.916 \\
Saturated solution & 1.096 & $2.22 \times 10^{-2}$ & 0.991 \\
Freeze-drying & 1.156 & $1.84 \times 10^{-2}$ & 0.971 \\
Spray-drying & 0.945 & $7.27 \times 10^{-3}$ & 0.956
\end{tabular}

The preparation by grinding was a relatively simple mixing process. ${ }^{36}$ A large amount of LCEO was not actually encapsulated into the microcapsule. Therefore, the release rate of microcapsules was similar to LCEO, which was a basic constant-speed process. The contents of LCEO in the microcapsules prepared by saturated aqueous solution or freezedrying were lower and decreased with the release of LCEO. The release rate of the microcapsules was reduced, which was consistent with the results reported by Wei Lei. ${ }^{37}$ The main resistance to the release of this kind of microcapsule was the density of the microcapsule shell material. ${ }^{38}$ The microcapsules produced using freeze-drying retained good solid skeletons, ${ }^{39}$ so the density of the shell material was stronger than that of the microcapsules prepared by saturated aqueous solution. ${ }^{40}$ Therefore, its protection of LCEO was slightly stronger than the latter. During the release process of microcapsules prepared by spray-drying, ${ }^{\mathbf{4 1}}$ the release environment volume was much larger than that of the microcapsules, and the size and surface area of the microcapsules remained unchanged, and the content of LCEO in the microcapsules was higher, so that the concentration of LCEO in the microcapsules might be approximately unchanged. ${ }^{\mathbf{4 2 , 4 3}}$ Therefore, the steady-state diffusion of LCEO in microcapsule film was an ideal diffusion process, which provided better protection for LCEO and greatly improved the stability of LCEO.

\section{Conclusions}

LCEO microcapsules were prepared using $\beta$-CD as shell material. The encapsulation rate of LCEO was the highest for spray-drying, followed by freeze-drying, saturated aqueous solution and grinding. Infrared spectrum analysis also verified this result. According to the SEM diagrams, the shape of the microcapsules prepared by spray-drying is more uniform than that of the other three methods. Spray-drying had the most satisfactory protective effect on the citral in LCEO, followed freeze-drying and saturated aqueous solution, while the grinding method appeared to have the worst protective effect under the same external conditions. Avrami's model was used to simulate the release rates of four kinds of microcapsules. It was found through an analysis of the release mechanism parameters and the release rate constant that the release reaction of the microcapsules prepared by grinding or spraydrying lay between the diffusion limiting kinetics and the first-order release kinetics, the release reaction of microcapsules prepared by saturated aqueous solution or freeze-drying was larger than the first-order release kinetics.

The release of the microcapsules was a complicated process. This theoretical study of the rate control of the release process, to some extent, revealed the release law of essentials oil in inclusion compounds. The study might also be used as a reference for other plant essential oils.

\section{Conflicts of interest}

There are no conflicts to declare.

\section{Acknowledgements}

We thank National Key R\&D Program of China (2017YFD0600704, P. R. China) and Open Foundation of Forestry Engineering discipline of Central South University of Forestry and Technology (P. R. China) for the financial support. 


\section{References}

1 C. Y. He, Yunnan Chem. Technol., 2005, 32, 1-7.

2 X. H. Chen, Mod. Chem. Ind., 2002, 22(7), 8-12.

3 X. Z. Li, J. Zhou and G. E. Yang, J. Chin. Cereals Oils Assoc., 2015, 30, 61-65.

4 R. P. Shen, S. P. Hu and X. H. Song, J. Chem. Eng. Chin. Univ., 2011, 25, 538-542.

5 C. B. Silva, S. S. Guterres and V. Weisheimer, Braz. J. Infect. Dis., 2008, 12, 63-66.

6 J. K. Hwang, E. M. Choi and J. H. Lee, Fitoterapia, 2005, 76, 684-686.

7 G. L. Andrea Yazmin, D. A. Lidia and J. F. Eugenia, J. Food Eng., 2012, 112, 29-37.

8 H. Y. Li, Y. J. Ma, Z. K. Li, J. Ji, Y. J. Zhu and H. Y. Wang, RSC Adv., 2017, 7, 50328-50335.

9 M. Homar, R. Dreu and J. Kerc, J. Microencapsulation, 2009, 26, 479-484.

10 D. A. Maria A., R. I. Sara and S. Torrado, J. Drug Targeting, 2006, 14(8), 567-575.

11 X. L. Yang, Y. Yuan and Y. R. Tan, Food Sci., 2013, 34, 359368.

12 A. Martin, S. Varona and A. Narrete, Open Chem. Eng. J., 2010, 4, 31-41.

13 A. P. Martinss, A. A. Craveiro, M. I. L Machado and J. Therm, Anal. Calorim., 2007, 88, 363-371.

14 A. Z. Fernando and G. A. Gustavo, J. Food Sci., 2010, 75, 398405.

15 B. Carmen, E. Andoni and I. M. Juan, LWT-Food Sci. Technol., 2015, 64, 1362-1369.

16 T. Metz, T. Haque and H. M. Chen, Drug Delivery, 2006, 13, 331-337.

17 A. Bahar, S. F. Coelho, F. J. Teles, P. C. Grijo, L. Francesca, C. A. Alberto, C. Attilio and P. Patrizia, LWT-Food Sci. Technol., 2018, 93, 220-228.

18 L. L. Han, L. W. Bi and Z. D. Zhao, Biomass Chem. Eng., 2011, 45, 41-46.

19 J. F. Shi, C. Yang and S. H. Zhang, ACS Appl. Mater. Interfaces, 2013, 5, 9991-9997.

20 M. M. Zuo, T. Q. Liu and J. J. Han, ACS Appl. Mater. Interfaces, 2014, 5, 6060-6067.

21 H. M. Zhu, Y. J. Zhang and F. Xu, Food Sci., 2017, 38, 106111.
22 B. Solomon, F. F. Sahle and T. Gebre, Eur. J. Pharm. Biopharm., 2012, 80, 61-66.

23 W. Ting, L. Bin and H. Y. Si, J. Inclusion Phenom. Macrocyclic Chem., 2011, 71, 207-213.

24 T. Koupantsis and A. Paraskevopoulou, Food Hydrocolloids, 2017, 69, 459-465.

25 L. Vora, V. G. Sita and P. Vavia, Eur. J. Pharm. Sci., 2017, 107, 78-86.

26 Y. Chen, M. Shu, X. X. Yao, K. Wu, K. Zhang, Y. T. He, K. Nishinari, G. O. Phillips, X. L. Yao and F. T. Jiang, Food Hydrocolloids, 2018, 84, 330-336.

27 D. Cristian, P. Livia, C. Alina, A. Petru and D. Stefan, Food Chem., 2016, 195, 39-48.

28 O. Ajda, I. Katja, S. Mihaela, S. Natasa, Z. Nada, K. Ksenija and U. N. Poklar, J. Food Eng., 2018, 229, 21-31.

29 M. Mai, J. M. Rosenholm, N. Prabhakar, M. Peurla, E. Baydoun and D. Patra, RSC Adv., 2015, 5, 18740-18750.

30 N. S. Sambudi, S. Cho and K. Cho, RSC Adv., 2016, 6, 4304143048.

31 T. Shi, P. Li and T. Shu, Sci. Technol. Food Ind., 2016, 37, 307311.

32 Q. Shen, Y. Zhao and Y. J. Chi, Mod. Food Sci. Technol., 2015, 31, 147-152.

33 F. You, L. X. Huang and C. H. Zhang, Chem. Ind. For. Prod., 2015, 35, 58-64.

34 N. N. Masoud, K. Rassoul and A. M. Seyed, Food Biophys., 2011, 6, 68-76.

35 Y. Wang, Z. T. Jiang and R. Li, Eur. Food Res. Technol., 2009, 228, 865-873.

36 T. Koeda, Y. i. Wada, T. L. Neoh, T. Wada, T. Furuta and H. Yoshii, Food Sci. Technol. Res., 2014, 20, 529-535.

37 L. Wei, X. L. Liu and K. G. Wu, J. Chem. Eng. Chin. Univ., 2014, 28, 336-341.

38 S. Petr, K. Pavel and S. Vladimir, J. Agric. Food Chem., 2012, 60(16), 4111-4119.

39 Z. J. Yang, Z. B. Xiao and H. B. Ji, Flavour Fragrance J., 2015, 30, 179-187.

40 K. Miriana, A. Lizette and G. G. Helene, Carbohydr. Polym., 2016, 151, 1245-1250.

41 S. J. Wang, Y. Shi and L. P. Han, J. Food Eng., 2018, 231, 1-9.

42 B. N. Estevinho and F. Rocha, Drying Technol., 2017, 35, 1249-1257.

43 L. P. Zhao, D. Han and H. Xiong, Food Sci., 2010, 31, 96-99. 